\title{
Joint Spectrum Allocation and Relay Selection in Cellular Cognitive Radio Networks
}

\author{
Ke Lang, Yuan Wu, and Danny H.K. Tsang \\ Department of Electronic and Computer Engineering \\ Hong Kong University of Science and Technology \\ Email: langke@ust.hk, ecewuy@ust.hk, eetsang@ece.ust.hk
}

\begin{abstract}
Cognitive radio network (CRN) has been proposed in recent years to solve the spectrum scarcity problem by exploiting the existence of spectrum holes. One of the important issues in a cellular CRN is how to efficiently allocate primary user (PU) spectrums without causing harmful interference to PUs. In this paper, we present a cross-layer framework which jointly considers spectrum allocation and relay selection with the objective of maximizing the minimum traffic demand of secondary users (SUs) in a cellular CRN. Specifically, we consider (1) CRN tries to utilize PU spectrums even when the CRN cell is not outside the protection region of the $\mathrm{PU}$ cell, and (2) cooperative relay is used in cellular CRNs to improve the utilization of PU spectrums. We formulate this cross-layer design problem as a mixed integer linear programming (MILP), and use LINGO to obtain the optimal solution. Compared with a simple channel allocation scheme, the numerical results show a significant improvement by using our method.
\end{abstract}

\section{INTRODUCTION}

Recent studies have shown that traditional fixed spectrum allocation policy is becoming inadequate to address today's rapid growing wireless communications. On the one hand, because of the increase in spectrum demand, this policy is facing spectrum scarcity problem. However, on the other hand, measurement studies indicate that a large portion of the assigned spectrums are not used in certain geographical areas in a significant duration of time. These two reasons render the traditional static spectrum allocation scheme insufficient, and hence a new technology called cognitive radio (CR) [1] is proposed to solve the spectrum scarcity and inefficiency problem.

Cooperative relay [2] could help improve spectrum utilization as well as the network throughput performance. Some research works [3] [4] [5] adopted cooperative relay in multihop ad hoc CRNs and studied the related joint spectrum and flow allocation problems. Some practical cognitive radio network like IEEE 802.22 system focuses on a cellular network which mainly aims to utilize DTV idle spectrums. Although channel allocation [6] and cooperative relay [2] [7] in cellular networks have been discussed widely, spectrum allocation in cellular CRNs still faces a special characteristic. i.e., CRNs have to opportunistically use the idle spectrum left by PUs and essentially have no fixed spectrum to use. This characteristic makes the incumbent protection (i.e., PU protection) very important in the successful application in CRNs.

[8] [9] used cooperative relay to improve the system throughput in cellular CRNs. However, in [8] [9] CRNs mainly used the so-called white space [10] of PU spectrum, and thus SUs' harmful co-channel interference to PUs' receivers was not explicitly considered.

In order to further enhance the system throughput, CRNs can aggressively operate on the so-called grey space [10] of PU spectrum by making use of space diversity. For example, even if CRN is inside the protection region of PU cells, it can still use the PUs' channel as long as the caused interference is unharmful (i.e., below some threshold).

Based on this consideration, in this paper, we study the joint spectrum allocation and relay selection problem for CRNs. We focus on the infrastructure-based CRNs (e.g., the model proposed in IEEE802.22). Specifically, we consider the following two special points in this work. First, we use both white space and grey space of PU spectrum available to enhance the CRNs throughput. To make use of the grey space, CRNs carry out a strict transmission power control policy so that PUs' receivers are sufficiently protected. Second, we use cooperative relay in CRNs to fully utilize the available spectrum resources (including both white and grey spaces of PU spectrum). Simulation results show that by making use of the above two points, CRN can improve its system performance significantly compared to that without using them.

The rest of the paper is organized as follows. We present the network model in section II. We then formulate the joint spectrum allocation and relay selection problem as a MILP in section III. Section IV shows the numerical results. Finally, we present some future work and conclude this paper in section V.

\section{SYSTEM MOdEL}

\section{A. Centralized Cognitive Radio Network Model}

We consider an infrastructure-based point to multiple point CRN model [11] [12] in this paper. The CRN base station (BS) is in charge of the uplink and downlink transmission for each SU in the CRN cell. Specifically, we assume that CRN BS knows the location of each SU as well as the location of each PU BS nearby. We consider a relatively static scenario where the PU spectrum environment is slow changing (e.g., DTV spectrum). For the PU network, only the PU BS is a transmitter, and all the PUs are just receivers (e.g., DTV system). 
In this work, we adopt two methods to improve the spectrum utilization of the CRNs. First, we adopt cooperative relay scheme to enhance spectrum reuse and system throughput performance. Second, in addition to the white space of PU spectrum, we also use the so-called grey space of PU spectrum ${ }^{1}$ to further improve the spectrum utilization.

Since PUs (e.g., DTV receivers) could be anywhere in the PU BS's transmission range and CRN BS could not obtain their location information, we adopt a conservative interference constraint in this paper, which means if CRN BS or SU intends to use the grey spectrum to transmit, then it should not have a downlink interference to the potential closest PU and thus not interfering with any potential PU.

We assume that each SU has multiple radio interfaces for data transmission, and all the transmitters' (i.e. including both PU and SU) transmission range and interference range are circular. In this work, we only consider the downlink case, and for the uplink case, the problem could be formulated in a similar way.

Fig. 1 is used to illustrate our ideas. Without our schemes and only using the conventional method, BS1 in CRN cell 1 and BS2 in CRN cell 2 can only transmit to SUs over idle PU channels directly. In comparison, using our ideas, Fig. 1 shows that in CRN cell 1, idle channels ch2 and ch3 are reused to enhance system performance. Besides, BS1 is using busy channel ch4 to transmit data to SU4 while limiting its transmission power so that the nearest PU receiver (i.e., the PU connected by a red dash dot line) is outside the interference range of this transmission. Similar case happens in CRN cell 2, SU1 relays some traffic to SU2 over busy channel ch7, and in this case, this transmission must also guarantee that it will not affect the nearest potential PU receiver.

In this work, we only consider intra-cell spectrum sharing problem. We assume inter-cell spectrum sharing use the exclusive allocation model [13], which means different CRN cells use different spectrum bands. Therefore, there is no interference among CRN cells.

In the rest of this paper, we focus on the spectrum allocation and relay selection problem in a single CRN cell. Table I lists the notations used in this paper.

\section{B. Signal Propagation Model and Link Capacity Formula}

There are two kinds of SUs in a CRN. The SUs with downlink traffic demand which we call destination nodes and the SUs without downlink traffic demand. Let $R$ denote the set of all SUs (excluding CRN BS) co-existing in CRNs. We further define the following two sets of SUs: (1) set $D$ denotes the set of SU nodes which have downlink traffic demands, and (2) we consider that each SU could be a potential relay, then the set including all SU nodes which can serve as relay is $R$.

\footnotetext{
${ }^{1}$ White space of PU spectrum refers to the PU spectrum which is currently not occupied by PUs, while grey space of PU spectrum, according to [10], is the spectrum that is currently partially occupied by PUs. Specifically in this work, if channel $b$ is grey space, it means some PU BS is currently using channel $b$. However, CRN BS can sense channel $b$ 's busy state, and opportunistically reuse channel $b$ in some area of the CRN cell as long as its reuse will not cause any harmful interference to PU BS.
}

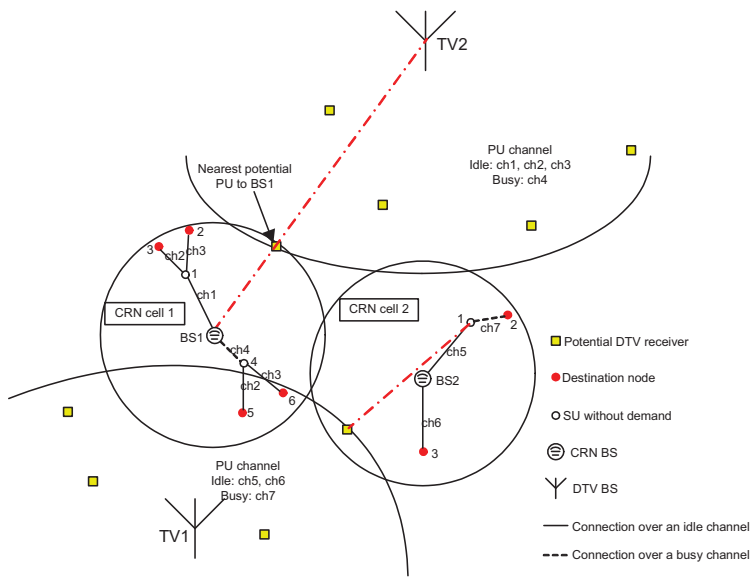

Fig. 1. An illustrated example of our ideas

TABLE I

NOTATIONS

\begin{tabular}{cc}
\hline Symbol & Descriptions \\
\hline $\mathcal{I}$ & Set of idle channels \\
$\mathcal{B}$ & Set of busy channels \\
$D$ & Set of SU destination nodes \\
$R$ & Set of all SUs \\
$W^{i}$ & Bandwidth of idle channel $i$ \\
$W^{b}$ & Bandwidth of busy channel $b$ \\
$P_{m}^{i}$ & Transmission power of m over idle channel $i$ \\
$P_{m}^{b}$ & Transmission power of m over busy channel $b$ \\
$P_{T V}^{b}$ & Transmission power of PU BS over busy channel $b$ \\
$G_{m}$ & Antenna gain of $m$ \\
$G_{T V}$ & Transmission antenna gain of PU BS \\
$h_{m, n}^{i}$ & Power propagation gain of link $(m, n)$ over idle channel $i$ \\
$h_{m, n}^{b}$ & Power propagation gain of link $(m, n)$ over busy channel $b$ \\
$h_{T V, n}^{b}$ & Power propagation gain of link (TV, $n$ ) over busy channel $b$ \\
$s_{m, n}$ & Distance between transmitter $m$ and receiver $n$ \\
$B_{m, n}^{i}$ & Capacity of link $(m, n)$ over idle channel $i$ \\
$B_{m, n}^{b}$ & Capacity of link $(m, n)$ over busy channel $b$ \\
$\alpha$ & Signal detection threshold over an idle channel \\
$\beta$ & Signal detection threshold over a busy channel \\
$t_{T V}^{b}$ & Transmission range of PU BS over busy channel $b$ \\
$t_{m}^{i}$ & Transmission range of m over idle channel $i$ \\
$t_{m}^{b}$ & Transmission range of m over busy channel $b$ \\
$t_{m, m a x}$ & Physical transmission range limit of $m$ \\
$T_{m}^{i}$ & Interference range of m over idle channel $i$ \\
$T_{m}^{b}$ & Interference range of m over busy channel $b$ \\
$\Delta$ & Guard zone \\
$\eta$ & Power of background noise \\
$N^{b}$ &
\end{tabular}

We only consider two-hop relay in this work and the multi-hop case will be briefly discussed in the future work.

As mentioned before, we want to utilize two kinds of spectrum spaces (i.e. white space of PU spectrum and grey space of PU spectrum). These two kinds of spectrum spaces refer to two kinds of PU channels. White space of PU spectrum refers to the idle channels (i.e., no PU is using this channel). Grey space of PU spectrum refers to the busy channels (i.e., some PU is currently using this channel, but SU can still use it 
as long as its transmission will not cause harmful interference to PU). We also define two sets of channels. Let $\mathcal{I}$ denote the set of idle channels, and let $\mathcal{B}$ denote the set of busy channels.

Let $W^{i}$ and $W^{b}$ denote the bandwidth of idle channel $i$ $(i \in \mathcal{I})$ and busy channel $b(b \in \mathcal{B})$, and let $B_{B S, d}^{i}, B_{B S, r}^{i}, B_{r, d}^{i}$ denote the capacity of link from CRN BS to destination node $d(d \in D)$, CRN BS to relay node $r(r \in R)$, and relay node $r$ to destination node $d$ over idle channel $i(i \in \mathcal{I})$ respectively. Each link capacity can be obtained by

$$
B_{m, n}^{i}=W^{i} \log _{2}\left(1+\frac{P_{m}^{i} G_{m} G_{n} h_{m, n}^{i}}{N}\right), \quad \forall m, n, i
$$

where $m \in R \cup\{B S\}, n \in R(m \neq n)$, and $h_{m, n}^{i}=s_{m, n}^{-\eta}$ $\left(s_{m, n}\right.$ is the distance between transmitter $m$ and receiver $n$ ) is the power propagation gain ${ }^{2} . P_{m}^{i}$ could be $P_{B S}^{i}$ (i.e., transmission power of the $\mathrm{CRN}$ BS) or $P_{r}^{i}$ (i.e. transmission power of SU r). $G_{m}, G_{n}$ denote the antenna gains of the transmitter and receiver, respectively. For example, when $m=B S, n=r, G_{m}$ is the antenna gain of the CRN BS (i.e., $G_{B S}$ ) and $G_{n}$ is the antenna gain of SU $r$ (i.e., $\left.G_{S U^{3}}{ }^{3}\right) . N$ is the power of the background noise. For example, $B_{B S, d}^{i}=W^{i} \log _{2}\left(1+\frac{P_{B S}^{i} G_{B S} G_{d} h_{B S, d}^{i}}{N}\right)$

Meanwhile, let $B_{B S, d}^{b}, B_{B S, r}^{b}, B_{r, d}^{b}$ denote the capacity of link from CRN BS to destination node $d(d \in D)$, CRN BS to relay node $r(r \in R)$, and relay node $r$ to destination node $d$ over busy channel $b(b \in \mathcal{B})$ respectively. We obtain the capacity for each link,

$$
B_{m, n}^{b}=W^{b} \log _{2}\left(1+\frac{P_{m}^{b} G_{m} G_{n} h_{m, n}^{b}}{N+P_{T V}^{b} G_{T V} G_{n} h_{T V, n}^{b}}\right), \forall m, n, b
$$

where $m \in R \cup\{B S\}, n \in R(m \neq n)$, and $P_{T V}^{b}$ is the DTV BS's transmission power, $G_{T V}$ is the DTV BS's antenna gain. $h_{m, n}^{b}=s_{m, n}^{-\eta}, h_{T V, n}^{b}$ (i.e., $s_{T V, n}^{-\eta}$ ) could be $h_{T V, d}^{b}$ (i.e., the power propagation gain from DTV BS to SU $d$ ) or $h_{T V, r}^{b}$ (i.e., the power propagation gain from DTV BS to $\mathrm{SU} r$ ). For example, $B_{B S, d}^{b}=W^{b} \log _{2}\left(1+\frac{P_{B S}^{b} G_{B S} G_{d} h_{B S, d}^{b}}{N+P_{T V}^{b} G_{T V} G_{d} h_{T V, d}^{b}}\right)$.

In this paper, we assume all SUs using idle channel have the same signal detection power threshold denoted by $\alpha$. Based on the successful transmission condition [4], we have

$$
P_{m}^{i} G_{m} G_{n} s_{m, n}^{-\eta} \geq \alpha
$$

We also assume all SUs using busy channel have the same signal detection power threshold denoted by $\beta$ which is larger than $\alpha$, because in using the busy channels, the noise not only contains background noise but also noises from the nearby DTV BS. Therefore, we have

$$
P_{m}^{b} G_{m} G_{n} s_{m, n}^{-\eta} \geq \beta
$$

From equations (3) and (4), the transmission range of $m$ ( $m \in R \cup\{B S\})$ over an idle channel $i(i \in \mathcal{I})$ denoted

\footnotetext{
${ }^{2}$ Specifically, $h_{m, n}^{i}=s_{m, n}^{-\eta} \rho_{m, n}^{i}$, where $s_{m, n}^{-\eta}$ is the path loss of link $(\mathrm{m}, \mathrm{n})$ with exponent $\eta$ (we set $\eta=4$ ) and $\rho_{m, n}^{i}$ is a normalized random factor modeling the frequency selective fading across different channels. In this work, we set $\rho_{m, n}^{i}=1$ for simplicity.

${ }^{3}$ In this work, we assume all SUs have the same antenna gain $G_{S U}$.
}

by $t_{m}^{i}$ (i.e. maximum $s_{m, n}$ with the guarantee of a successful transmission) can be expressed as:

$$
t_{m}^{i}=\left(\frac{P_{m}^{i} G_{m} G_{S U}}{\alpha}\right)^{1 / \eta}
$$

Meanwhile, the transmission range of the BS and SU over a busy channel can be expressed as:

$$
t_{m}^{b}=\left(\frac{P_{m}^{b} G_{m} G_{S U}}{\beta}\right)^{1 / \eta}
$$

By using equations (5) and (6), we can determine SU's transmission range from the transmission power. Conversely, we can also determine SU's transmission power from the transmission range. Since the transmission range is related to the interference range which will be discussed in the following, we can control the transmitter's power (to make its interference range small enough) to avoid any potential interference to PUs.

For clear presentation, we put (5) and (6) into (1) and (2) respectively. The capacity formula becomes a function of transmission range and the distance between transmitter and receiver as follows:

$$
\begin{aligned}
& B_{m, n}^{i}=W^{i} \log _{2}\left(1+\frac{\alpha}{N}\left(\frac{t_{m}^{i}}{s_{m, n}}\right)^{\eta}\right) \\
& B_{m, n}^{b}=W^{b} \log _{2}\left(1+\frac{\left(\frac{t_{m}^{b}}{s_{m, n}}\right)^{\eta}}{\frac{N}{\beta}+\left(\frac{t_{T V}^{b}}{s_{T V, n}}\right)^{\eta}}\right)
\end{aligned}
$$

In the following, we use equations (7) and (8) instead of the original capacity formulas (1) and (2).

\section{Protocol Interference Model}

In this work, we adopt the Protocol Interference Model [14]. The corresponding interference range denoted by $T_{m}^{i}, T_{m}^{b}$ can be obtained by the following equations:

$$
\begin{aligned}
& T_{m}^{i}=(1+\Delta) t_{m}^{i} \\
& T_{m}^{b}=(1+\Delta) t_{m}^{b}
\end{aligned}
$$

Since we adopt the conservative interference constraint and the locations of all SUs and PU BSs are known to the CRN BS, we can obtain the interference range limit for the CRN BS and each SU when using a busy channel. For the transmission over an idle channel $i$, since the transmitter could use its physical power limit, we have

$$
T_{m}^{i}=(1+\Delta) t_{m, \max }
$$

where $t_{m, \max }$ is the maximum transmission range of transmitter $m$.

However, for the transmission over a busy channel $b$, the transmitter may not use its physical power limit in order to protect PUs. In this case (shown in Fig. 1), we have

$$
T_{m}^{b}=\min \left\{(1+\Delta) t_{m, \max }, d_{T V, m}-t_{T V}\right\}
$$

where $d_{T V, m}-t_{T V}$ is the distance from the transmitter to the potential nearest PU (distance from PU BS to the transmitter minus the PU BS's transmission range $t_{T V}$ which is shown as the red dash dot line in Fig. 1). 
Since the locations of CRN BS and all SUs are fixed, the interference ranges of CRN BS and all SUs which can be obtained from (11) and (12) are also fixed. From (9) and (10), the transmission ranges of CRN BS and all SUs could be obtained. Therefore, from (5) and (6) the transmission powers of CRN BS and all SUs could be adjusted.

\section{PROBLEM Formulation}

\section{A. Link Assignment and Flow Assignment}

The decision variables representing channel allocation and relay selection decisions are:

$$
\begin{array}{r}
x_{B S, d}^{i}, x_{B S, r}^{i}, x_{r, d}^{i}, x_{B S, d}^{b}, x_{B S, r}^{b}, x_{r, d}^{b} \in\{0,1\}, \\
\forall d \in D, r \in R, i \in \mathcal{I}, b \in \mathcal{B}
\end{array}
$$

where for example, $x_{B S, d}^{i}=0$ denotes CRN BS not allocating channel $i$ for link $(B S, d)$, while $x_{B S, d}^{i}=1$ denotes CRN BS allocating channel $i$ for link $(B S, d)$.

Meanwhile. the decision variables representing flow rate allocation for each link over each channel are:

$$
\begin{array}{r}
f_{B S, d}^{i}, f_{B S, r}^{i}, f_{r, d}^{i}, f_{B S, d}^{b}, f_{B S, r}^{b}, f_{r, d}^{b} \geq 0, \\
\forall d \in D, \quad r \in R, i \in \mathcal{I}, \quad b \in \mathcal{B}
\end{array}
$$

where for example, $f_{B S, d}^{i}$ denotes the flow rate allocation for link $(B S, d)$ over channel $i$. Notice that $f_{B S, d}^{i}=0$ means that CRN doesn't allocate any rate for link $(B S, d)$ on channel $i$.

\section{B. Transmission Range Constraint}

As described before, careful channel allocation and relay selection should be carried out by CRN BS so that PUs only suffer unharmful interference. To achieve this, we need to limit the transmission range of the CRN BS as well as the SUs when using busy channel to transmit. This means CRN BS and SUs have to use smaller transmission power instead of their physical power limits when using busy channels.

When the locations of CRN BS, PU BS, and all SUs are fixed, the interference ranges of CRN BS (i.e., $T_{B S}^{i}, T_{B S}^{b}$ ) and SUs (i.e., $T_{S U}^{i}, T_{S U}^{b}$ ) are also fixed.

Specifically, we consider the following two cases:

Case 1: allocation of the channel $i(i \in \mathcal{I})$. For the idle channel transmission range limit and interference range limit, they are just the ranges when using the physical power limit because this transmission will not cause interference to the PUs. Therefore, CRN BS could transmit to any SUs in the cell over an idle channel, and SU could relay traffic to the SUs which are within its physical transmission range limit (i.e. $t_{m}^{i}=t_{m, \max }, m \in R \cup\{B S\}$ ), .

Case 2: allocation of the channel $b(b \in \mathcal{B})$. When using busy channels, both the CRN BS and the SUs may need to transmit lower than their physical power limits in order to protect PUs. From equations (11) and (12), we can obtain the transmission range of CRN BS and SUs as $t_{m}^{b}=T_{m}^{b} /(1+$ $\Delta), m \in R \cup\{B S\}$.

Based on the transmission ranges, we know that the link assignment should be 0 when the receiver is outside the transmission range of the transmitter. In summary, we can express the set of constraints regarding to the channel allocation mathematically as follows:

$$
\begin{gathered}
x_{r, d}^{i}=0, \forall d \in D, r \in R, i \in \mathcal{I}, s_{r, d}>t_{r, \max } \\
x_{B S, d}^{b}=0, \forall d \in D, r \in R, b \in \mathcal{B}, s_{B S, d}>t_{B S}^{b} \\
x_{B S, r}^{b}=0, \forall d \in D, r \in R, b \in \mathcal{B}, s_{B S, r}>t_{B S}^{b} \\
x_{r, d}^{b}=0, \forall d \in D, r \in R, b \in \mathcal{B}, s_{r, d}>t_{r}^{b}
\end{gathered}
$$

\section{Mutual Interference Constraint}

Besides the interference to PU, mutual (co-channel) interference should be avoided in CRN. Therefore, we have the following constraints for the idle channels:

$$
\begin{array}{r}
\sum_{r \in R} \sum_{d \in D}\left(x_{B S, d}^{i}+x_{B S, r}^{i}\right) \leq 1, \forall i \in \mathcal{I} \\
x_{B S, d}^{i}+x_{r, d^{\prime}}^{i} \leq 1, \forall i \in \mathcal{I}, \forall r \in R, \forall d, d^{\prime} \in D \\
x_{B S, r}^{i}+x_{r^{\prime}, d}^{i} \leq 1, \forall i \in \mathcal{I}, \forall r, r^{\prime} \in R, \forall d \in D \\
x_{r, d}^{i}+x_{r^{\prime}, d^{\prime}}^{i} \leq 1, \quad \forall d, d^{\prime} \in D, r, r^{\prime} \in R, i \in \mathcal{I}, \\
(r, d) \neq\left(r^{\prime}, d^{\prime}\right), s_{r, d^{\prime}} \leq T_{r}^{i} \\
x_{r, d}^{i}+x_{r^{\prime}, d^{\prime}}^{i} \leq 1, \quad \forall d, d^{\prime} \in D, r, r^{\prime} \in R, i \in \mathcal{I}, \\
(r, d) \neq\left(r^{\prime}, d^{\prime}\right), s_{r^{\prime}, d} \leq T_{r^{\prime}}^{i}
\end{array}
$$

In (22) and (23) $(r, d) \neq\left(r^{\prime}, d^{\prime}\right)$ means the constraints are applied only when links $(r, d)$ and $\left(r^{\prime}, d^{\prime}\right)$ are two different links, and the scenario that two links overlap at only one point applies the constraints. Specifically, when $d=d^{\prime}, r \neq r^{\prime}$, the constraints avoid two relay nodes transmit to the same destination node using the same channel. When $r=r^{\prime}, d \neq d^{\prime}$, the constraints avoid one relay node transmit to two different destination nodes over the same channel. When $r \neq r^{\prime}, d \neq d^{\prime}$, the constraints avoid two links within each other's interference range use the same channel to transmit.

For the scenario of using nearby PU cell busy channels, the constraints are similar but due to the decreased BS's power and relay node's power, the interference ranges shrink, and we have the following constraints for the busy channels:

$$
\begin{gathered}
\sum_{r \in R} \sum_{d \in D}\left(x_{B S, d}^{b}+x_{B S, r}^{b}\right) \leq 1, \forall b \in \mathcal{B} \\
x_{B S, d}^{b}+x_{r, d^{\prime}}^{b} \leq 1, \forall d, d^{\prime} \in D, r \in R, b \in \mathcal{B}, s_{B S, d^{\prime}} \leq T_{B S}^{b} \\
x_{B S, d}^{b}+x_{r, d^{\prime}}^{b} \leq 1, \forall d, d^{\prime} \in D, r \in R, b \in \mathcal{B}, s_{r, d} \leq T_{r}^{b} \\
x_{B S, r}^{b}+x_{r^{\prime}, d}^{b} \leq 1, \forall d \in D, r, r^{\prime} \in R, b \in \mathcal{B}, s_{r^{\prime}, r} \leq T_{r^{\prime}}^{b} \\
x_{B S, r}^{b}+x_{r^{\prime}, d}^{b} \leq 1, \quad \forall d \in D, r, r^{\prime} \in R, b \in \mathcal{B}, s_{B S, d} \leq T_{B S}^{b} \\
x_{r, d}^{b}+x_{r^{\prime}, d^{\prime}}^{b} \leq 1, \quad \forall d, d^{\prime} \in D, r, r^{\prime} \in R, b \in \mathcal{B}, \\
(r, d) \neq\left(r^{\prime}, d^{\prime}\right), s_{r, d^{\prime}} \leq T_{r}^{b} \\
x_{r, d}^{b}+x_{r^{\prime}, d^{\prime}}^{b} \leq 1, \quad \forall d, d^{\prime} \in D, r, r^{\prime} \in R, b \in \mathcal{B}, \\
(r, d) \neq\left(r^{\prime}, d^{\prime}\right), s_{r^{\prime}, d} \leq T_{r^{\prime}}^{b}
\end{gathered}
$$




\section{Flow Conservation and Capacity Constraint}

To impose certain fairness criteria on each destination node rate demand in the CRN, we set the objective function as maximizing the scaling factor $\lambda$ for all the destination node rate requirements. Through this, we actually achieve a maxmin fairness over all SUs' traffic demands. Then, we have

$\sum_{i \in \mathcal{I}} \sum_{b \in \mathcal{B}} \sum_{r \in R}\left(f_{B S, d}^{i}+f_{r, d}^{i}+f_{B S, d}^{b}+f_{r, d}^{b}\right) \geq \lambda \cdot \gamma(d), \quad \forall d \in D$

where $\gamma(d)$ denotes destination node $d$ 's traffic demand. Equation (31) means all traffic finally come to destination node $d$ should be larger than or equal to some proportion of $d$ 's traffic demand.

Besides, we have the flow conservation constraints to guarantee the total incoming flow and the total outgoing flow of any relay node to be equal. Therefore,

$\sum_{i \in \mathcal{I}} f_{B S, r}^{i}+\sum_{b \in \mathcal{B}} f_{B S, r}^{b}=\sum_{d \in D} \sum_{i \in \mathcal{I}} f_{r, d}^{i}+\sum_{d \in D} \sum_{b \in \mathcal{B}} f_{r, d}^{b}, \quad \forall r \in R$

The flow rate allocation and channel allocation are coupled by the following constraints.

$$
\begin{gathered}
f_{B S, d}^{i} \leq x_{B S, d}^{i} B_{B S, d}^{i}, \quad \forall d \in D, i \in \mathcal{I} \\
f_{B S, r}^{i} \leq x_{B S, r}^{i} B_{B S, r}^{i}, \quad \forall r \in R, i \in \mathcal{I} \\
f_{r, d}^{i} \leq x_{r, d}^{i} B_{r, d}^{i}, \quad \forall d \in D, \quad r \in R, \quad i \in \mathcal{I} \\
f_{B S, d}^{b} \leq x_{B S, d}^{b} B_{B S, d}^{b}, \quad \forall d \in D, b \in \mathcal{B} \\
f_{B S, r}^{b} \leq x_{B S, r}^{b} B_{B S, r}^{b}, \quad \forall r \in R, b \in \mathcal{B} \\
f_{r, d}^{b} \leq x_{r, d}^{b} B_{r, d}^{b}, \quad \forall d \in D, r \in R, \quad b \in \mathcal{B}
\end{gathered}
$$

For example, constraint (33) states that the flow allocation for link $(B S, d)$ on channel $i$ cannot exceed the link capacity according to the channel allocation decision $x_{B S, d}^{i}$.

\section{E. Problem formulation}

The complete problem is formulated as follows:

$$
\max \lambda
$$

subject to:

$$
(13)-(38)
$$

The formulated problem is a MILP problem, which is very difficult to solve in general. We use LINGO (an optimization software) to find the optimization solution, which we denote by "Binary X" scheme in the following.

\section{F. A Relaxation Approach}

The channel allocation scheme used in section (III-E) means that CRNs can only decide whether or not to allocate a specific channel to a specific link.

However, a more flexible scheme is that CRNs can further decide how much transmission time should be allocated within a frame so that the links can time-share a channel. Following is an example considering the proposed IEEE 802.22 framework. According to [11] each MAC superframe contains 16 frames and each frame contains an integer number of MAC slots. Therefore, CRN BS can further determine the percentage of the transmission time to allocate channels in a frame.

Our previous problem formulation can be smoothly extended to include this feature.

Specifically, we relax the constraint that $x_{B S, d}^{i}$ should be chosen from a binary value (i.e. $x_{B S, d}^{i} \in\{0,1\}$ ) to the constraint that $x_{B S, d}^{i}$ can now be chosen from any real value within $[0,1]$ (i.e. $\left.x_{B S . d}^{i} \in[0,1]\right)^{4}$. All decision variables representing the channel allocation for the link from relay nodes to destination nodes remain as binary values. Mathematically, we change constraint (13) into:

$$
\begin{array}{r}
x_{B S, d}^{i}, x_{B S, r}^{i}, x_{B S, d}^{b}, x_{B S, r}^{b} \in[0,1], \text { and } \\
x_{r, d}^{i}, x_{r, d}^{b} \in\{0,1\}, \forall d \in D, r \in R, i \in \mathcal{I}, b \in \mathcal{B}
\end{array}
$$

The formulation of this relaxation approach is as follows:

$$
\max \lambda
$$

subject to:

$$
(14)-(39)
$$

We denote the optimal solution of this relaxed problem by "Relaxed BS X" scheme.

\section{NumERICAL RESUlts}

In this section, we present numerical results for our proposed methods and compare them with a simple channel allocation scheme.

\section{A. Parameter Settings}

Table II shows the transmission range of CRN BS and PU BS (coverage of CRN cell and PU cell), and the transmission range from a SU to another SU. Table III and table IV shows the topology of the PU BS, CRN BS, all SUs and the destination SUs' demands. Table V shows the value of the parameters used in our experiments.

\section{B. Numerical Results}

Fig. 2 shows that using "Binary X" scheme, the channel allocation and relay selection results. It is shown that CRN BS tends to use busy channels in a direction away from the PU cell which is reasonable because this will cause less interference to PUs. Meanwhile idle channels 1 and 2 are reused in this SU cell to get the best system performance.

${ }^{4}$ Notice that in practical application, these values should be discrete values instead of real values. Therefore, for practical application, we can approximate the real values into close discrete values. As long as there are many slots in each frame, the performance using real values will be close to the practical case. 
TABLE II

TRANSMISSION RANGE LIMIT

\begin{tabular}{cc}
\hline Type of user & Physical transmission range limit \\
\hline CR BS & $33 \mathrm{~km}$ \\
DTV BS & $132 \mathrm{~km}$ \\
SU to SU & $15 \mathrm{~km}$ \\
\hline
\end{tabular}

TABLE III

LOCATION OF SU AND PU BSS

\begin{tabular}{cc}
\hline Type of user & Location \\
\hline 802.22 BS & $(0,0)$ \\
DTV BS & $(0,165)$ \\
\hline
\end{tabular}

TABLE IV

SUS' LOCATION OF A RANDOM TOPOLOGY AND THEIR DEMANDS

\begin{tabular}{ccc}
\hline SU's node index & Location & Demand \\
\hline 1 & $(12,12)$ & 0 \\
2 & $(-11,28)$ & $2 \mathrm{Mbps}$ \\
3 & $(-12,20)$ & 0 \\
4 & $(-19,26)$ & $2 \mathrm{Mbps}$ \\
5 & $(-13,1)$ & $2 \mathrm{Mbps}$ \\
6 & $(-17,-19)$ & 0 \\
7 & $(10,-10)$ & 0 \\
8 & $(5,-18)$ & 0 \\
9 & $(9,-20)$ & $2 \mathrm{Mbps}$ \\
10 & $(20,-18)$ & $2 \mathrm{Mbps}$ \\
\hline
\end{tabular}

TABLE V

PARAMETERS

\begin{tabular}{cc}
\hline Symbol & Value \\
\hline $\mathcal{I}$ & ch1, ch2, ch3 \\
$\mathcal{B}$ & ch4, ch5 \\
$D$ & $2,4,5,9,10$ \\
$R$ & $1-10$ \\
$W^{i}$ & $1 \mathrm{MHz}$ \\
$W^{b}$ & $1 \mathrm{MHz}$ \\
$\Delta$ & 1 \\
$\eta$ & 4 \\
$\alpha$ & $N$ \\
$\beta$ & $1.2 N$ \\
\hline
\end{tabular}
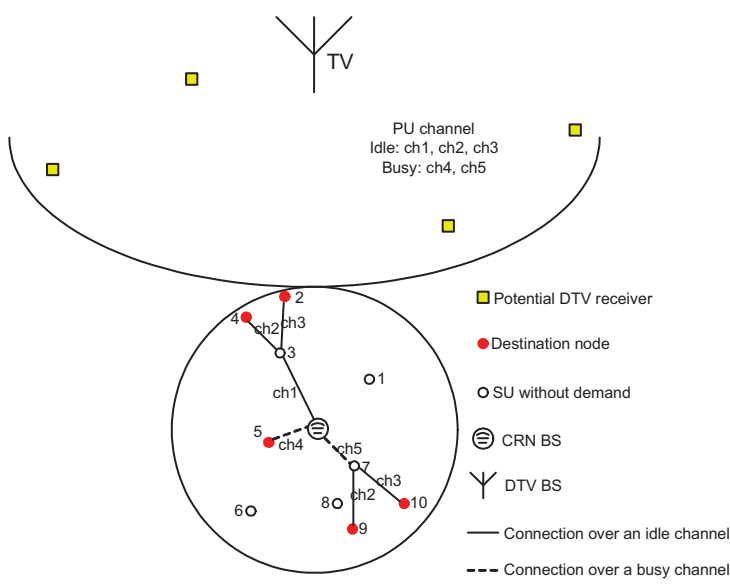

Fig. 2. "Binary $X "$ scheme with $|\mathcal{I}|=3$, and $|\mathcal{B}|=2$
One frame at BS

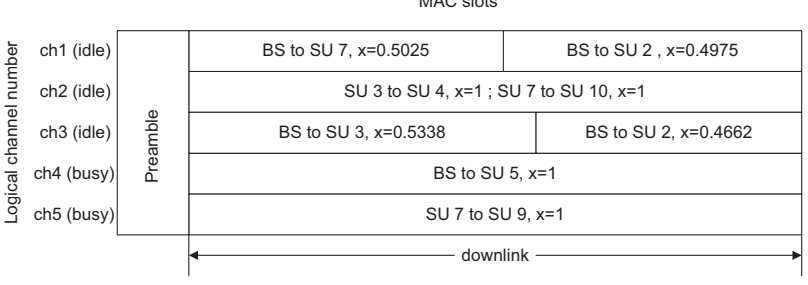

Fig. 3. "Relaxed BS X" scheme with $|\mathcal{I}|=3$, and $|\mathcal{B}|=2$

Fig. 3 shows that using the "Relaxed BS X" scheme, the results of the channel allocation and relay selection. It is shown from Fig. 3 that this scheme gives the CR BS more flexibility to allocate channels in a frame.

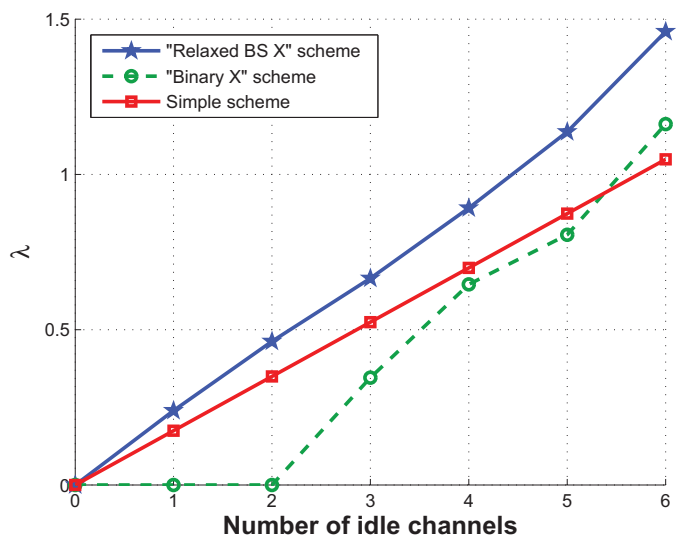

Fig. 4. Comparison of $\lambda$ with different number of idle channels when $|\mathcal{B}|=2$

For comparison, we also consider a simple scheme here. Specifically, the simple scheme is just maximizing fairness factor $\lambda$ without using relay and busy channels but with relaxed channel allocation variable $x_{B S, d}^{i}(d \in D, i \in \mathcal{I})$ from $[0,1]$. This means it is just allocating idle channels to make the destination nodes achieve even percentage of their demands without the help of relay scheme and grey space of PU spectrum.

It is shown in Fig. 4 that our scheme ("Relaxed BS $X$ " scheme) has a significant improvement over the simple scheme ${ }^{5}$ even only with 2 logical busy channels. With the increase of idle channels (all the other parameters remain the same and only the number of idle channels changes), the improvement by our scheme becomes better and better than the simple method. The "Binary X" scheme is not good because of its inflexibility in allocating channels for multiple MAC

${ }^{5}$ Notice that in this work, we assume that the PU spectrums are slow changing and the PU is not coming back in the middle of a CRN MAC frame. However, in reality, the PU may come back in the middle of a CRN MAC frame. In that case, both the performance of our schemes and the performance of the simple scheme should be slightly decreased. This situation also exists in the following results. 
slots in one frame.

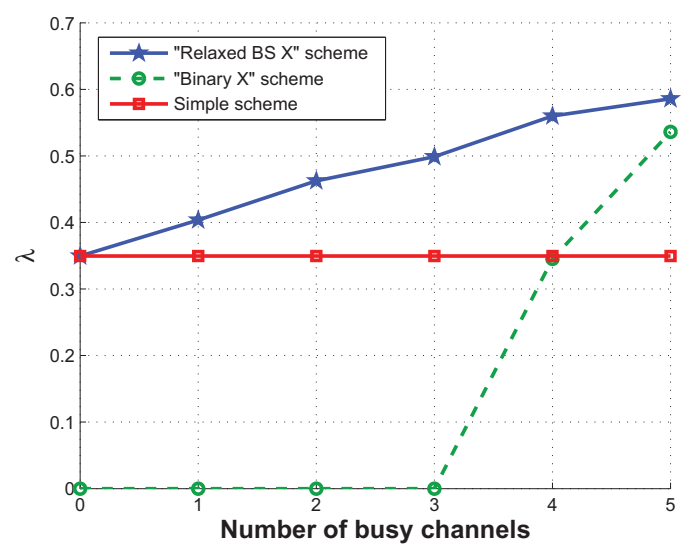

Fig. 5. Comparison of $\lambda$ with different number of busy channels when $|\mathcal{I}|$ $=2$

Fig. 5 shows the performance of our proposed schemes have similar trends as Fig. 4 when the number of busy channels increases (all the other parameters remain the same, only the number of idle channels is set as 2 and the number of busy channels changes). However, the increase of $\lambda$ is much slower than in Fig. 4 because when using busy channels, CRN BS and SUs need to consider the interference to the nearby PU cell and thus they need to decrease their transmission power. However, when using idle channels, CRN BS and SUs need not to consider the interference to PU.

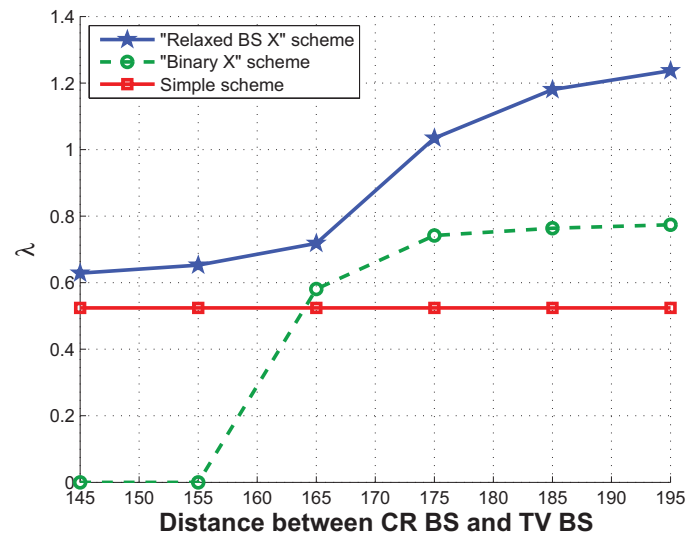

Fig. 6. Comparison of $\lambda$ with different distance between CR BS and TV BS when $|\mathcal{I}|=3,|\mathcal{B}|=3$

Fig. 6 shows the performance comparison when the distance between PU BS and CRN BS changes. It shows that when the distance between PU BS and CRN BS increases, the performance of our proposed schemes becomes better because CRN BS and SUs could transmit using more power over those busy channels of the nearby PU cell. We notice that when the distance between PU BS and CRN BS is very close, and in some cases the CRN cell could be even included in the PU cell, then the improvement of the "Relaxed BS $X$ " scheme over the simple method may just come from the advantage of relay. When the distance between PU BS and CRN BS becomes larger, the performance increase of the "Relaxed BS X" scheme becomes smaller. Finally when the CRN cell is outside the keep-out region [12] of the PU cell, the performance of the "Relaxed BS X" scheme stops increasing. Because at this time, the CRN cell can fully utilize the spectrums in the PU cell, and thus those busy channels becomes idle channels for the CRN cell.

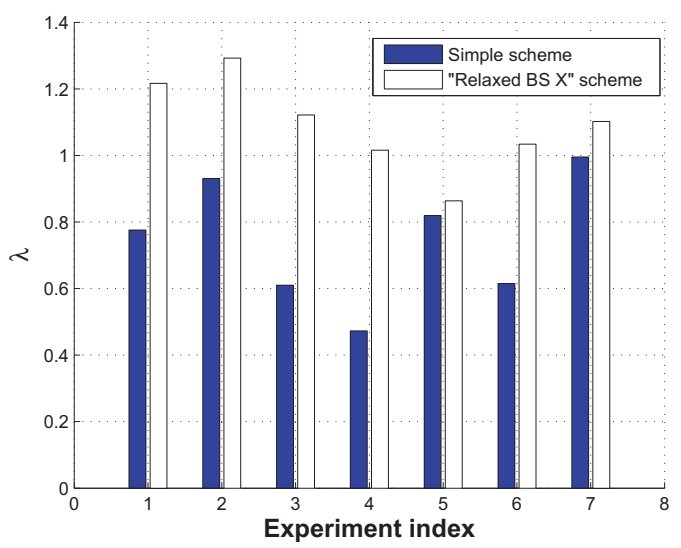

Fig. 7. Comparison of $\lambda$ in multiple experiments with randomly generated topologies

Fig. 7 shows the comparison of $\lambda$ between using our proposed "Relaxed BS X" scheme and simple scheme in multiple experiments. In each experiment, we set different random topologies (i.e. locations of the PU BS, CRN BS and all SUs) as well as different SUs' traffic demands. In this figure, it is shown that in most of the experiments, the improvement by our proposed scheme is significant. In some experiments (e.g. experiments 5 and 7), the improvements seem not that good because in these cases, the CRN cell is very close to the PU cell or even is covered by the PU cell, and thus, CRN BS and SUs cannot utilize the grey spectrums in the PU cell. Besides, in these two cases, due to the bad location of SUs, relay cannot make a good influence to improve the overall performance.

\section{CONClusion And Future Work}

In this paper, we present an optimization framework which maximizes the fairness factor in a cellular CRN while jointly considers spectrum allocation and relay selection. We formulate it as a MILP problem and use LINGO to obtain the optimal solution. Compared with a simple channel allocation scheme, the numerical results show a significant improvement by using our method.

Currently, we only focus on the intra-cell spectrum allocation and relay selection problem. In our future work, we will 
extend this problem to the inter-cell case. For instance, the CRN cells with the fairness factor $\lambda<1$ (i.e., SUs' demands cannot be fully satisfied) may initiate inter-cell spectrum sharing requests, and those CRN cells with $\lambda>1$ (i.e., SUs' demands can be fully satisfied) could lend some of their spectrums to the neighbor CRN cells having inter-cell spectrum sharing requests so that their own demands can still be satisfied while at the same time their neighbor CRN cells could have a better performance.

\section{REFERENCES}

[1] I. F. Akyildiz, W. Y. Lee, M. C. Vuran, S. Mohanty, "Next generation/dynamic spectrum access/cognitive radio wireless networks: A survey," Computer Networks (Elsevier) vol. 50, no. 13, pp. 2127-2159, May 2006.

[2] J. Cho and Z. J. Haas, "On the Throughput Enhancement of the Downstream Channel in Cellular Radio Networks Through Multihop Relaying," IEEE Journal On Selected Areas In Communications, vol. 22, no. 7, pp. 1206-1219, September 2004

[3] Y. T. Hou, Y. Shi, and H. D. Sherali, "Spectrum Sharing for Multi-Hop Networking with Cognitive Radios," IEEE Journal On Selected Areas In Communications, vol. 26, no. 1, pp. 146-155, January 2008

[4] M. Ma and D. H. K. Tsang, "Joint Design of Spectrum Sharing and Routing with Channel Heterogeneity in Cognitive Radio Networks," Physical Communication, volume 2, issues 1-2, pp. 127-137, March-June 2009

[5] Y. Wu and D. H. K. Tsang, "Dynamic Rate Allocation, Routing and Spectrum Sharing for Multi-Hop Cognitive Radio Networks," in Proc. of IEEE International Conference on Communications Workshops, 2009, pp. 1-6, June 14-18, 2009

[6] I. Katzela and M. Naghshineh, "Channel Assignment Schemes for Cellular Mobile Telecommunication Systems: A Comprehensive Survey," IEEE Personal Communications, pp. 10-31, June, 1996

[7] Y. Liu, R. Hoshyar, X. Yang, and R. Tafazolli, 'Integrated Radio Resource Allocation for Multihop Cellular Networks With Fixed Relay Stations," IEEE Journal On Selected Areas In Communications, vol. 24, no.11, pp. 2137-2146, November 2006

[8] J. Jia, J. Zhang and Q. Zhang, "Cooperative Relay for Cognitive Radio Networks," in Proc. IEEE INFOCOM 2009, pp. 2304-2312, April 2009

[9] Q. Zhang, J. Jia and J. Zhang, "Cooperative Relay to Improve Diversity in Cognitive Radio Networks," IEEE Communications Magazine, pp. 111117, February 2009

[10] S. Haykin, "Cognitive Radio: Brain-Empowered Wireless Communications," IEEE Journal On Selected Areas In Communications, vol. 23, no. 2, pp. 201-220, February 2005

[11] IEEE 802.22 WG, "Draft standard for wireless regional area networks part 22: Cognitive wireless RAN medium access control (MAC) and physical layer (PHY) specifications: Policies and procedures for operation in the TV bands," IEEE P802.22/D0.3.8.1, September 2007.

[12] C. Cordeiro, K. Challapali, and D. Birru, "IEEE 802.22: An Introduction to the First Wireless Standard based on Cognitive Radios," IEEE Journal of Communications, vol. 1, no. 1, pp. 38-47, April 2006.

[13] W. Y. Lee and I. F. Akyildiz, "Joint Spectrum and Power Allocation for Inter-Cell Spectrum Sharing in Cognitive Radio Networks," in Proc. IEEE DYSPAN 2008, pp. 1-12, October 2008

[14] P. Gupta and P. R. Kumar, "The capacity of wireless networks," IEEE Transactions on Information Theory, vol. 46, no. 2, March 2000 